\title{
State of art and advances on the treatment of bone metastases from breast cancer: a concise review
}

\author{
Lorenzo Rossi $^{1,2}$, Claudio Longhitano ${ }^{1,2}$, Florenc Kola ${ }^{3}$, Maria Del Grande ${ }^{1}$ \\ ${ }^{1}$ Institute of Oncology of Southern Switzerland (IOSI), Bellinzona, Switzerland; ${ }^{2}$ Breast Unit of Southern Switzerland (CSSI), Lugano, Switzerland; \\ ${ }^{3}$ Department of Internal Medicine, Ente Ospedaliero Cantonale, Bellinzona, Switzerland \\ Contributions: (I) Conception and design: L Rossi; (II) Administrative support: L Rossi, M Del Grande; (III) Provision of study materials or patients: \\ All authors; (IV) Collection and assembly of data: L Rossi, F Kola, M Del Grande; (V) Data analysis and interpretation: L Rossi, M Del Grande; (VI) \\ Manuscript writing: All authors; (VII) Final approval of manuscript: All authors. \\ Correspondence to: Lorenzo Rossi. Institute of Oncology of Southern Switzerland (IOSI), Bellinzona, Switzerland; Breast Unit of Southern Switzerland \\ (CSSI), Lugano, Switzerland; Ospedale San Giovanni, Bellinzona, Switzerland. Email: lorenzo.rossi@eoc.ch.
}

\begin{abstract}
Bone is one of the most common metastatic sites in metastatic breast cancer (mBC). The presence of bone metastases can lead to various complications including pain, spinal cord compression, hypercalcaemia, pathological fractures. The treatment of bone metastases of breast cancer (BC) is mainly based on the biological characteristics of the primary tumour, but there are also specific treatments for bone lesions including bone modifiers, radionuclides, or localized treatments such as radiotherapy, surgery or vertebroplasty. In this review, we analyse the state of art of the common treatments and the advances of bone metastases of BC. Finally, we summarize the state of art of biomarkers in diagnosis, prognosis and prediction to therapy.
\end{abstract}

Keywords: Breast cancer (BC); bone metastases; bisphosphonates; denosumab; biomarkers

Submitted Aug 10, 2019. Accepted for publication Jan 14, 2020.

doi: $10.21037 /$ cco.2020.01.07

View this article at: http://dx.doi.org/10.21037/cco.2020.01.07

\section{Introduction}

Incidence of breast cancer (BC) is growing every year (1). Metastatic breast cancer $(\mathrm{mBC})$ counts approximately 6-10\% of new cases (2) ("de novo" metastatic disease), and it is estimated that $20 \%$ to $30 \%$ of all BC will develop metastases. Bone metastases (BM) count approximately $60-70 \%$ of all $\mathrm{mBC}(3,4)$ and more than $70 \%$ of patients showed bone metastases during autopsy (5). Among the most important complications of BM there are pain, pathological fractures, spinal compression and hypercalcemia.

This complex of events is often included in the definition of skeletal-related events (SREs), in which the need for radiotherapy or surgery for pain or spinal cord decompression is also considered. In mBC, SREs occur in about half to two thirds of patients with bone metastases (6). SREs remain a major cause of mortality and morbidity, and has an impact on the quality of life (7). Furthermore, the occurrence of an SRE influences the median 5-year survival rate, reducing it from $8.3 \%$ to $2.5 \%$ (6). Therefore, reducing the risk of SRE is a priority objective in these patients, and it is recommended to administrate bonemodifying agents (BMAs) before symptomatic events (8-10). The purpose of this short review is to describe the state of the art of BM treatments, including systemic and locoregional treatment of the bone. Finally, we will briefly discuss the current state of the art of promising biomarkers for the diagnosis and monitoring of BM.

\section{Systemic treatments}

\section{Antitumor treatments}

The choice of systemic therapy should be based on the biological characteristics of metastatic lesions. It would be 
preferable to obtain these biological characteristics from a biopsy of the metastatic lesions rather than infer them based on the characteristics of the primitive tumour, except for "de novo" mBC (11), due to the possible discrepancy in hormone receptor status and human epidermal receptor growth factor (HER2) status between primary BC and metastasis (12). Alongside systemic anticancer therapy, in presence of $\mathrm{BM}$, there are several therapies focused on the treatment of bone lesions, such as bone modifiers or radionuclides.

\section{Bone modifiers}

Currently, in clinical practice bisphosphonates and denosumab are the two targeted-drugs most used in the treatment of BC BM.

Bisphosphonates act on the inhibition of protein prenylation by blocking the osteoclast farnesyl phyrophosphate synthase. The final effect is the inhibition of bone resorption (13).

The most common bisphosphonates in clinical practice for the treatment of $\mathrm{BC} B M$ are pamidronate and zoledronic acid.

In vitro, bisphosphonates shown to be able to inhibit tumour adhesion and neo-angiogenesis and to induce tumour apoptosis (14). In vivo, there are some evidences that bisphosphonates can reduce tumour burden also in extraskeletal environment, such as liver and lung parenchyma, with a dose-dependent mechanism (15).

The action of bisphosphonates, and particularly of zoledronic acid, appears to be synergistic with doxorubicin chemotherapy. In fact, when zoledronic acid is administered 24 hours after doxorubicin, there is an up to 10 -fold increase in tumour apoptosis. The most accredited hypothesis on these observations is a stronger uptake of zoledronic acid in chemotherapy-sensitized cells, leading to a greater tendency to arrest the cell cycle. These data has been recently confirmed by the ANZAC trial, a phase II trial that explored the activity of zoledronic acid given 24 hours after 5 -fluorouracil-epirubicin-cyclophosphamide in neoadjuvant setting (16). Despite the interesting results shown in this trial, there were no important implications for dose scheduling in clinical practice on neoadjuvant setting.

Starting about 20 years ago, at least three trials evaluated the role of bisphosphonates in the $\mathrm{BC}$ adjuvant setting (17-19). The primary endpoints of these three trials were incidence of distant metastases (skeletal and non-skeletal), and overall survival (OS). The results were discordant and this discrepancy can be explained by heterogeneity of study population.

In the ABCSG-12 trial (20), 1,803 premenopausal patients with stage I/II luminal BC randomized to receive tamoxifen or anastrozole plus goserelin with or without zoledronic acid every 6 months $(4 \mathrm{mg})$, for 3 years. The primary endpoints were disease-free survival (DFS) and recurrence-free survival (RFS). After a median followup of 4 years, there was an improvement in DFS of $36 \%$ in zoledronic acid arm $(\mathrm{P}=0.01)$ and a trend in favour of zoledronic acid on OS, but without statistical significance (HR 0.60, 95\% CI: 0.32-1.11, P=0.11). Intriguingly, the benefit was not limited to skeletal events but it was also in terms of loco-regional and distant recurrence rates.

Two parallel trial, Z-FAST (21) and ZO-FAST (22), randomized a total of 2,195 postmenopausal patients with early BC to receive letrozole for 5 years and early (at start of study) or delayed (in case of $T$ score $\leq 2.0$ or osteoporotic fracture) zoledronic acid, respectively.

The primary endpoint was the change in lumbar spine bone density (BMD) at 12 months. Secondary endpoints were the change on lumbar spine and total hip BMD in the subsequent follow-up, DFS and OS. After 60 months of follow-up, there was a significant benefit in terms of BMD change on early zoledronic acid $(+4.3 \%$ vs. $-5.4 \%$, $\mathrm{P}<0.0001)$. There was a benefit also in DFS in favour of early zolendronate group (relative risk reduction of $41 \%$, absolute difference 3.2\%, $\mathrm{P}=0.0314$ ). As in ABCSG-12 trial, the benefit was in both local and distant recurrence $(0.4 \%$ vs. $1.9 \%$ and $3.8 \%$ vs. $5.6 \%$, respectively). Finally, AZURE trial (23) randomized 3,360 patients to receive neoadjuvant chemo- or endocrine therapy with or without zolendronate in neoadjuvant (every 3-4 weeks for six administrations) and adjuvant (three-monthly for eight administrations and then 6-monthly for five administrations) setting, consecutively. The primary endpoint was the comparison of DFS. After a median follow-up of 59 months, there were no differences in DFS between the two arms (HR 0.98, $\mathrm{P}=0.79)$ (23). Intriguingly, in the subgroup of women in postmenopausal status for more than five years, there was an improvement on OS (HR 0.71, $\mathrm{P}=0.017$ ). This can explain the differences between the results of the AZURE trial and the ABCSG-12 trial, in which all enrolled patients received ovarian suppression with goserelin. A possible hypothesis could be that bisphosphonates act better with low oestrogen levels (24).

The NSABP-B34 trial (25) (clodronate vs. placebo for three years in adjuvant setting) was a multicentre, randomised, double-blind, placebo-controlled trial 
conducted on 3,323 patients with early (stage I-III) BC. Patients were randomly assigned to receive after surgery either oral clodronate $1,600 \mathrm{mg}$ daily for 3 years or placebo. The primary endpoint was DFS rate. DFS did not differ between the two arms (286 events in experimental arm versus 312 in placebo arm, HR 0.91, 95\% CI: 0.778-1.07, $\mathrm{P}=0.27$ ), and there was no difference even in OS (HR 0.84, 95\% CI: 0.67-1.05, $\mathrm{P}=0.13$ ).

However, looking only at women over the age of 50 , in the experimental arm there was a statistically significant increase in DFS (HR 0.75, 95\% CI: 0.57-0.99, P=0.045), in the time without bone metastases (HR 0.62, 95\% CI: 0.40 0.95, $\mathrm{P}=0.027$ ), but not in OS (HR 0.80, 95\% CI: 0.61-1.04, $\mathrm{P}=0.094)(25)$.

Authors reported a suboptimal rate of non-adherence in the clodronate group (44\% of withdrawals from treatment), possibly due to gastrointestinal side-effects. In our opinion, this is a major problem of the trial that can limit the value of its results. Furthermore, 23 patients in the experimental group and 12 in the placebo group experienced hepatic dysfunction $\geq$ grade 3 . The authors also described a single case of osteonecrosis of the jaw in the experimental group. The limited magnitude of this study does not allow definitive conclusions to be drawn.

Final results from the SUCCESS trial (NCT02181101adjuvant zolendronate for two versus five years) are awaited and could further clarify the role of bisphosphonates in the adjuvant setting as prevention of BM.

In metastatic setting, no trials demonstrated an impact of bisphosphonates in OS, but they could reduce the risk of skeletal events, reduce the bone pain and improve the quality of life $(4,26)$. The efficacy of bisphosphonates to reduce the risk of SRE has been confirmed by a Cochrane analysis in which bisphosphonates was compared with placebo or no treatment in patients with BC BM. The reduction of the SRE risk was $14 \%$, and there was a reduction of bone pain (4).

Bisphosphonates can also exert some indirect antitumor effects in the bone milieu, as suggested by some preclinical studies.

In the bone marrow microenvironment, a possible sanctuary for disseminated tumor cells that can evade systemic anticancer therapies and lay dormant for long periods, bisphosphonates might alter the levels of several growth factors $(27,28)$.

Moreover, bisphosphonates reduce skeletal tumor burden in several preclinical models of bone metastasis from different solid tumors, including BC. This reduction can be attributed mainly to the antiresorptive activity of these drugs (29).

In particular, an interesting preclinical study (30) showed that an analogue of risedronate (NE-58051) with a 3,000fold lower bone antiresorptive activity, do not inhibit $\mathrm{BC} \mathrm{BM}$ cells or bone tumor burden, suggesting that the antitumoral activity of bisphosphonates is mainly due to inhibition of osteoclast-mediated bone resorption.

A retrospective analysis of phase III trials using zolendronate showed that in patients with elevated levels NTX (N-telopeptide of type I collagen), a bone-resorption marker, the normalization during 3 months of zoledronate correlates with an improvement on OS (9 months) (31). Another possible explanation could be that the survival benefit can be derived from the reduction of skeletal morbidity (such as fractures) rather than a direct anticancer effect.

The optimal frequency of bisphosphonates administration is still debated and has been investigated by several trials. Among these, we want to mention two trials that directly compared a 12 -week schedule with a 4-week schedule. The OPTIMIZE-2 trial was a prospective, randomized, doubleblind, multicenter phase 3 trial in which 416 women were randomized to receive zolendronate every 4 weeks or every 12 weeks, while 13 patients received placebo. The primary endpoint was the SRE rate. Secondary endpoints included time to first SRE and skeletal morbidity rate. After 1 year of follow-up, there was a difference in events of $1.2 \%$ (22\% in the 4-week arm, $23.2 \%$ in the 12 -week arm, non-inferiority $\mathrm{P}=0.02)$. The time to first $\mathrm{SRE}$ was not statistically different (HR 1.06, 95\% CI: 0.70-1.60, P=0.79). The safety profile was comparable between the two arms (32). ZOOM trial was a non-inferiority, phase 3 , multicenter trial that randomized 425 patients with BC BM with completed 12-15 months of monthly zolendronate to receive zolendronate $(4 \mathrm{mg})$ every 12 or every 4 weeks. The primary endpoint was SRE rate. After one year of follow-up, the difference between the two arms was 4\% (26\% in 12 -week arm, $22 \%$ in 4 -week arm). The Authors concluded that the 12 -week schedule was non-inferior to the 4-week schedule (33). From a cost-effective point-of-view, the 12 -week schedule is a reasonable alternative to the 4-week schedule (34). Actually, the guidelines of the National Comprehensive Cancer Network (NCCN) (26) and ESMO (9) recommend to switch to a 12 -week schedule after 1 year of monthly treatment with zolendronate. The duration of the treatment with bisphosphonate is not clear, and it depends of tolerability and toxicity. 


\section{Page 4 of 11}

Denosumab is an IgG2 monoclonal antibody that inhibits the RANKL-RANK interaction binding to receptor activator of nuclear factor k-B ligand (RANK) and preventing osteoclast formation and survival (35). Like bisphosphonates, denosumab has also been explored in the adjuvant setting of $\mathrm{BC}$ as a potentially preventive drug for $\mathrm{BM}$, with discordant results. The ABCSG-18 trial randomized 1,711 postmenopausal patients with early luminal $\mathrm{BC}$ to receive denosumab 60 mg every 6 months or placebo. The primary endpoint was the rate of clinical fractures. Results showed a significant reduction of clinical fractures (11.15 with denosumab, $26.2 \%$ with placebo, HR $0.50,95 \%$ CI: $0.30-0.65)(36)$, and a modest improvement in DFS in the denosumab arm (5-year DFS: 89.25 with denosumab vs. 87.35 with placebo, HR 0.82, 95\% CI: 0.69-0.98, absolute gain $1.9 \%$ at 5 years and $3.15 \%$ at 8 years) (37). Conversely, D-CARE trial (38) found no positive effect on either its primary endpoint of BM free-survival or DFS, the secondary endpoint. In particular, D-CARE trial randomized 4,509 pre and post-menopausal patients with non-metastatic BC with high risk of recurrence (nodal involvement at diagnosis, tumour size $>5 \mathrm{~cm}$ or locally advanced disease) to receive monthly denosumab 120 $\mathrm{mg}$ for six doses or placebo. After a median follow-up of 67 months there were no significant differences in BM free survival or DFS (HR 0.97 and 1.04 respectively, with a nonsignificant $\mathrm{P}$ value). Moreover, no subgroup has benefit from denosumab. The study population of the D-CARE trial was more homogeneous and significant different from the ABCSG-18 trial, in which $75 \%$ of patients received chemotherapy in early setting and $100 \%$ received aromatase inhibitors. This could partly explain the differences in results between the two trials. Data on role of denosumab in adjuvant setting is still uncertain and bisphosphonates should remain the standard of care.

In metastatic setting, the comparison between denosumab and zoledronate has been investigated in a large trial that randomized 2,046 patients with BC BM (39). The trial showed an improvement in time to first onstudy SRE (HR 0.82; 95\% CI: 0.71-0.95, $\mathrm{P}<0.001$ noninferiority; $\mathrm{P}=0.01$ superiority) and in reducing risk of multiple SRE $(\mathrm{P}=0.001)$ in denosumab arm with a dose of $120 \mathrm{mg}$ monthly. OS was the same between arms (HR 0.95; 95\% CI: 0.81-1.11, P=0.49). The optimal duration of the BC BM treatment with denosumab is not still clarified. Generally, the use of denosumab in clinical trial has been evaluated up to two years or until unacceptable toxicity.
Rossi et al. The treatment of bone metastases from breast cancer

As with zoledronic acid, schedules with different dosing intervals were also explored for denosumab, in order to reduce side effects and maintain the same efficacy. The SAKK 96/12 trial (NCT02051218) is currently in progress, with the aim to test the hypothesis that the administration for 12 weeks is not inferior to administration every 4 weeks in terms of first on-trial SSE. The estimated enrollment is 1,380 patients with $\mathrm{BC}$ or prostate cancer, and the estimated study competition date is December 2022. Preliminary data published in the form of abstracts and presented in the San Antonio Breast Cancer Symposium 2018 (40) show that denosumab-related hypocalcemia is less frequent with administration every 12 weeks.

The side effects of bone-modifiers such as bisphosphonates and denosumab are similar: osteonecrosis of jaw, nephrotoxicity and hypocalcemia are the most frequent. The incidence of osteonecrosis of jaw (ONJ) is $0.6 \%$ to 6.25 in patients with $\mathrm{BC} \mathrm{BM}$ treated with bisphosphonates and the incidence is similar in patients treated with denosumab (39). The most important risk factors for ONJ are inflammatory dental or alveolar diseases, long exposition to therapy with bone modifiers (41), glucocorticoid or anti-angiogenic therapies (42). The treatment with bisphosphonates or denosumab should be delayed for 2-3 weeks after invasive dental procedures (43). Nephrotoxicity is more frequent with zolendronate than with denosumab (39). Renal failure related to bisphosphonate is dose-depended. Dose adjustment should be evaluated according to the grade of renal impairment and the clearance of creatinine. In case of decrease of renal function during bisphosphonate therapy, drug should be withheld until renal function returns to within 10 percent baseline (43).

Hypocalcemia and hypophosphatemia are common side effects during treatment with denosumab $(39,44)$. Thus, calcium and vitamin $\mathrm{D}$ supplementation is recommended in order to prevent hypocalcemia. More rarely, bone modifiers can cause cardiac arrhythmias like atrial fibrillation and supraventricular tachycardia (45), skin reaction, ototoxicity (46) or infections (47).

\section{Radionuclides}

Systemic radionuclide therapy can be used to manage pain derived from BM after failure of systemic analgesics or radiotherapy, especially when the side effects correlated to high dose analgesics become intolerable.

Main contraindications are asymptomatic bone lesions, 
pure osteolytic lesions or inadequate marrow reserve, spinal cord compression, high risk of fracture or pathologic fracture of weight bearing bone, renal failure, pregnancy and breastfeeding (48). Furthermore, since radionuclides have an action latency from 1 to 4 weeks, patients' prognosis should be more than 3 months.

The general principle of functioning of radionuclides is deliver local radiation by emitting beta particles trying to avoid deep radiation of bone marrow. Radionuclides approved and commonly used in radionuclide therapy are Strontium-89 hydrochloride (Sr-89), samarium-153 lexidronam (Sm-153) and rhenium-186 hydroxyethylidenediphosphonate (Re186), while Phosphorus-32 (P-32) is outdated due to severe myelotoxicity.

Other clinical trials evaluating the palliation potential of other radionuclides such as tin $\left({ }^{117} \mathrm{mSn}\right)$ diethylenetriaminepentaacetate (DTPA), sodium $\left({ }^{33} \mathrm{P}\right)$ phosphate, rhenium $\left({ }^{188} \mathrm{Re}\right)$-hydroxyethylidenediphosphonate (HEDP), lutetium $\left({ }^{177} \mathrm{Lu}\right)$-EDTMP, and radium $\left({ }^{223} \mathrm{Ra}\right)-$ chloride are ongoing.

The most significant toxicity is characterized by transient myelosuppression with neutropenia, anemia and, less frequently, thrombocytopenia (48).

In patients with $\mathrm{BC} B M$, a clinical benefit rate in terms of pain reduction of $70-80 \%$ has been reported after 1 week of intravenous ${ }^{53} \mathrm{Sm}$-EDTMP administration with prolonged responses of up 12 months (49).

Serafini and colleagues conducted a comprehensive review of data about clinical response rate on systemic radionuclide therapy; in summary, the response rate for BC $\mathrm{BM}$ ranged from $50 \%$ to $92 \%(50)$, and these results were confirmed by other authors $(51,52)$.

Following the observation that response duration is longer in patients treated earlier with radionuclides, it was hypothesized that radiopharmaceuticals could play a role in eradicating micrometastatic disease localized in bone marrow (53). This tumoricidal effect appears to be enhanced when radiopharmaceuticals are administered in synergy with other treatments such as external-beam radiotherapy and/ or systemic therapy (chemotherapy or endocrine therapy) $(54,55)$. However, these approaches could be burdened with greater toxicity, especially haematological. The multimodality approach using bisphosphonate, chemotherapy and radiopharmaceutical has been shown to be superior in terms of symptoms control and prolonged survival rather than chemotherapy or radiopharmaceuticals alone (56).

Another strategy to increase the rate and duration of clinical responses is the administration of repeated doses of radionuclides. Kasalický and colleagues reported prolonged response duration in patients with $\mathrm{BC} \mathrm{BM}$ after receiving multiple ${ }^{89} \mathrm{SR}$ administrations compared with patients who had received a single administration $(3.08 \pm 0.48$ versus $5.33 \pm 2.36$ months) (57).

\section{New drugs}

In the last few decades, a large number of new molecules have been investigated as promising drugs for $\mathrm{BC} B M$ therapy. In vitro, Dasatinib, a tyrosine kinase used in chronic myeloid leukemia, has been seen as capable of inhibiting osteoclastogenesis (58). Its role on treatment of BM from BC has been evaluated on SWOG S0622 trial, a phase II clinical trial aimed to determine an improvement on PFS and a decrease of serum bone turnover markers (59). Seventy-nine patients with BM from BC were enrolled. After a median PFS of 12.6 weeks, there were no significant differences in PFS, without statistically significance $(\mathrm{P}=0.85)$ (59). Therefore, this trial failed to demonstrate an advantage of dasatinib in this cohort of patients, but further studies with wider accrual should be conducted.

In a phase 1 study, another tyrosine-kinase, saracatinib, was seen to reduce bone resorption markers up to $88 \%$ from baseline after $24 \mathrm{~h}$ with no effect on bone formation markers and without serious adverse events (60). These results are similar to results derived from previous trials on bisphosphonates in in women with BM from BC. Data on the clinical efficacy of saracatinib are still early and need further validation in phase 2 and 3 clinical trials.

Other promising agents are curcuminoids, which could prevent tumour growth-factor beta (TGF- $\beta$ ) induction of parathyroid hormone-related protein $(\mathrm{PTHrP})$ reducing osteolytic bone reabsorption (61). The efficacy of this class of drugs in reducing osteolysis resulting from $\mathrm{BM}$ of $\mathrm{BC}$ has been confirmed in in vivo murine models (62). In clinical setting, a phase I trial has been conducted to investigate the feasibility and safety of docetaxel and curcumin in patients with $\mathrm{BM}$ from $\mathrm{BC}(63)$. This trial enrolled 14 patients that received $6,000 \mathrm{mg}$ per day of curcumin, for 7 days every 3 weeks in combination with docetaxel. Preliminary data on efficacy are encouraging and a phase II clinical trial on curcumin plus docetaxel versus docetaxel singleagent recently terminated, but data are not yet published (NCT00852332).

Some monoclonal antibodies that block PTHrP or proteasome inhibitors that can directly interfere with the process of bone metastases by tumor cells are also 
promising.

An interesting new strategy is the combination of cytotoxic agents with antigen-specified nano-particles as vectors, e.g., micelle with $\alpha v \beta 3-M P$-docetaxel. This nanoparticle recognizes $\alpha v \beta 3$ integrin in $\mathrm{BC}$ cells in the bone and can carry the chemotherapeutic drug within the $\mathrm{BM}$ with less systemic toxicity (64).

Teriparatide is a bone-anabolic agent currently used in the treatment of osteoporosis. It is able to increase the bone formation and some authors have also reported a suppression of the growth of myeloma lesions in bone (65). The authors suggest that teriparatide can be effective also in solid tumor metastases, including BC.

Abaloparatide is a homologue of the parathyroid hormone (PTH) and the parathyroid hormone-related protein (PTHrP) (66). It is able to perform an anabolic action on the bone, while it has a limited effect on bone resorption. The exact pharmacokinetics and pharmacodynamics mechanisms of the abaloparatide are better described elsewhere (66). Its clinical use is currently limited to therapy of osteoporosis, however it is a promising agent for the treatment of hypercalcemia. To our knowledge, there are no ongoing trials on the role of abaloparatide in the treatment of BC BM.

\section{Loco-regional treatments}

In the case of risk or in the presence of a skeletal segment fracture, surgery is the first choice option, especially in the case of spinal compression, where the decompression treatment is a urgency. In case of impossibility or contraindication to perform surgery, vertebroplasty can be a good alternative to surgery for vertebral compression fractures. It is minimally invasive and there is clinical evidence for improvement in pain and physical function (67). Another therapeutic option is radiotherapy (RT) $(25,68)$, which also has a primary role in treating the pain of individual bone lesions when systemic pain therapy is not adequate. In this case, one single fraction with $8 \mathrm{~Gy}$ is recommended, because dose intensification or fractionated irradiation does not result in a better analgesic effect (69).

\section{Biomarkers}

The detailed description of the molecules and physiological or pathophysiological processes of normal or metastatic bone metabolism is beyond the scope of this review and is well described elsewhere (70).

Ad ideal biomarker of BC BM should have: a diagnostic role (in terms of detection of presence of BM), an early prognostic value (e.g., estimate the risk of a patient with early BC to develop BM) a late prognostic value (e.g., estimate the risk of $\mathrm{BM}$ progression or develop SRE) or a predictive value (e.g., estimate the probability of response to targeted treatments for $\mathrm{BM}$ ).

Some bone turnover markers (BTM) have been investigated as diagnostic markers of BC BM. For example, the tartrate resistant acid phosphatase (TRACP) and the pro-collagen type $1 \mathrm{~N}$-terminal pro-peptide (P1NP) were seen to increase significantly in the presence of $\mathrm{BM}$, especially when BMs were $>7$, with discordant results $(71,72)$. The receptor activator of nuclear factor KB-ligand/ osteoprotegerin ratio was also reported to be significantly increased in BC BM, with a sensitivity and specificity of $73 \%$ and $72 \%$, respectively (73). However, specificity, sensitivity and diagnostic efficacy are suboptimal and BTM do not yet have a diagnostic role in routine clinical practice, where imaging techniques are preferred.

Some BTMs such as serum bone alkaline phosphatase (sBALP) and urinary N-telopeptide of type 1 collagen (uNTX) have been investigated as prognostic markers. Coleman and colleagues (74) reported a significant correlation between sBALP and UNTX and the occurrence of SRE in 1,824 patients under zolendronate or pamidronate for BM from different malignancies. Similarly, Brown and colleagues (75) demonstrated a significant correlation between high sBALP and uNTX levels at baseline and the risk of SRE in patients under bisphosphonates. Conversely, in patients with high baseline levels of uNTX, the normalization of this value during the bisphosphonate treatment was significant correlated with less SRE or death risk (76). Moreover, in patients with NTX levels within the normal range throughout the treatment duration has a reduction $(40-68 \%)$ of the death or SRE risk $(\mathrm{P}<0.0005)$ as compared to those with raised NTX levels (77).

Few trials have investigated the potential role of BTM in predicting the efficacy of bone modifiers such as bisphosphonates or denosumab in BC BM. Most of the data derives from randomized clinical trials that included patients with different malignancies, including BC. Coleman and colleagues (78) reported that in patients with various malignancies or multiple myeloma and with baseline NTX >100 nmol/mmol creatinine, there was a significant reduction of death risk of $31 \%(\mathrm{P}=0.0028)$ if a zolendronate treatment was administrated. However, these results should be validated on larger populations with BC BM. Furthermore, some concomitant treatments or intrinsic 
features of the patients (e.g., age, sex, comorbidity etc.) as well as seasonal variations can interfere with the BTM levels influencing the evaluation $(70,79)$.

Several studies have tried to identify a significant genomic signature for increased risk of BC BM. A number of genes coding for membrane molecules involved in protein binding (80), or genes for interleukin 1-beta (IL-1ß) (81) or for interleukin 1 receptors (82), or genes coding for zinc-finger proteins (such as ZNF217) (83) were identified, all significantly related to a greater tumour cell osteotropism. However, these data derive exclusively from preclinical studies, and there are no significant validations in the clinical setting to date. In the analysis of breast tumour samples from 724 patients enrolled in the AZURE trial (mentioned above), high expression of macrophagecapping protein (CAPG) and PDZ domain-containing protein member 1 (GIPC1) was significantly correlated with the risk of development of $\mathrm{BM}(\mathrm{P}<0.001)$ and with the efficacy of zolendronate in preventing $\mathrm{BM}(\mathrm{P}=0.008)(84)$. Thus, the combined CAPG/GIPC1 biomarker is one of the most promising biomarkers of response to bisphosphonates, although further and larger studies are needed to validate its use in current clinical practice.

For a long time the CA 15-3 tumor marker was used as a biomarker of $\mathrm{mBC}$. It is not bone-specific and is not recommended as marker of surveillance due to low sensitivity (30\%) (85).

Currently there are no bone-specific biomarkers used in clinical practice (86). In fact, the American Society of Clinical Oncology guidelines do not recommend use of biochemical biomarkers as treatment monitoring, due to lack of strong evidence deriving from published studies (43).

\section{Conclusions}

The optimal management requires a multidisciplinary approach that includes medical and radiation therapy, orthopaedic surgery, interventional radiology, nuclear and palliative interventions to control symptoms, avoid SREs and improve quality of life.

\section{Acknowledgments}

Funding: None.

\section{Footnote}

Conflicts of Interest: All authors have completed the ICMJE uniform disclosure form (available at http://dx.doi. org/10.21037/cco.2020.01.07). The authors have no conflicts of interest to declare.

Ethical Statement: The authors are accountable for all aspects of the work in ensuring that questions related to the accuracy or integrity of any part of the work are appropriately investigated and resolved.

Open Access Statement: This is an Open Access article distributed in accordance with the Creative Commons Attribution-NonCommercial-NoDerivs 4.0 International License (CC BY-NC-ND 4.0), which permits the noncommercial replication and distribution of the article with the strict proviso that no changes or edits are made and the original work is properly cited (including links to both the formal publication through the relevant DOI and the license). See: https://creativecommons.org/licenses/by-nc-nd/4.0/.

\section{References}

1. Jemal A, Center MM, DeSantis C, et al. Global patterns of cancer incidence and mortality rates and trends. Cancer Epidemiol Biomarkers Prev 2010;19:1893-907.

2. Press DJ, Miller ME, Liederbach E, et al. De novo metastasis in breast cancer: occurrence and overall survival stratified by molecular subtype. Clin Exp Metastasis 2017;34:457-65.

3. Parkes A, Clifton K, Al-Awadhi A, et al. Characterization of bone only metastasis patients with respect to tumor subtypes. NPJ Breast Cancer 2018;4:2.

4. O'Carrigan B, Wong MH, Willson ML, et al. Bisphosphonates and other bone agents for breast cancer. Cochrane Database Syst Rev 2017;10:CD003474.

5. Coleman RE. Clinical features of metastatic bone disease and risk of skeletal morbidity. Clin Cancer Res 2004;10:4559-67.

6. Yong M, Jensen AO, Jacobsen JB, et al. Survival in breast cancer patients with bone metastases and skeletal-related events: a population-based cohort study in Denmark (1999-2007). Breast Cancer Res Treat 2011;129:495-503.

7. Weinfurt KP, Li Y, Castel LD, et al. The significance of skeletal-related events for the health-related quality of life of patients with metastatic prostate cancer. Ann Oncol 2005;16:579-84.

8. Wong MH, Stockler MR, Pavlakis N. Bisphosphonates and other bone agents for breast cancer. Cochrane Database Syst Rev 2012;2:CD003474. 


\section{Page 8 of 11}

9. Coleman R, Body JJ, Aapro M, et al. Bone health in cancer patients: ESMO Clinical Practice Guidelines. Ann Oncol 2014;25:iii124-37.

10. Mortimer J, Mendelsohn M. Improving the use of intravenous bisphosphonates in women with breast cancer metastatic to bone. J Natl Compr Canc Netw 2014;12:S40-1.

11. Sharma M, Gogia A, Deo SSV, et al. Role of rebiopsy in metastatic breast cancer at progression. Curr Probl Cancer 2019;43:438-42.

12. Curtit E, Nerich V, Mansi L, et al. Discordances in estrogen receptor status, progesterone receptor status, and HER2 status between primary breast cancer and metastasis. Oncologist 2013;18:667-74.

13. Kavanagh KL, Guo K, Dunford JE, et al. The molecular mechanism of nitrogen-containing bisphosphonates as antiosteoporosis drugs. Proc Natl Acad Sci U S A 2006;103:7829-34.

14. Winter MC, Holen I, Coleman RE. Exploring the antitumour activity of bisphosphonates in early breast cancer. Cancer Treat Rev 2008;34:453-75.

15. Hiraga T, Williams PJ, Ueda A, et al. Zoledronic acid inhibits visceral metastases in the 4T1/luc mouse breast cancer model. Clini Cancer Res 2004;10:4559-67.

16. Winter MC, Cross SS, Ingram CE, et al. ANZAC: a neoadjuvant biomarker study exploring the antitumour activity on the addition of Zoledronic Acid to Chemotherapy in breast cancer. Ann Oncol 2009;20:165P.

17. Diel IJ, Solomayer EF, Costa SD, et al. Reduction in new metastases in breast cancer with adjuvant clodronate treatment. N Engl J Med 1998;339:357-63.

18. Saarto T, Vehmanen L, Virkkunen P, et al. Ten-year follow-up of a randomized controlled trial of adjuvant clodronate treatment in node-positive breast cancer patients. Acta Oncol 2004;43:650-6.

19. Powles T, Paterson A, McCloskey E, et al. Reduction in bone relapse and improved survival with oral clodronate for adjuvant treatment of operable breast cancer (ISRCTN83688026). Breast Cancer Res 2006;8:R13.

20. Gnant M, Mlineritsch B, Schippinger W, et al. Endocrine therapy plus zoledronic acid in premenopausal breast cancer. N Engl J Med 2009;360:679-91.

21. Brufsky AM, Bosserman LD, Caradonna RR, et al. Zoledronic acid effectively prevents aromatase inhibitor-associated bone loss in postmenopausal women with early breast cancer receiving adjuvant letrozole: Z-FAST study 36-month followup results. Clin Breast Cancer 2009;9:77-85.

22. Eidtmann H, de Boer R, Bundred N, et al. Efficacy of
Rossi et al. The treatment of bone metastases from breast cancer

zoledronic acid in postmenopausal women with early breast cancer receiving adjuvant letrozole: 36-month results of the ZO-FAST Study. Ann Oncol 2010;21:2188-94.

23. Coleman R, Cameron D, Dodwell D, et al. Adjuvant zoledronic acid in patients with early breast cancer: final efficacy analysis of the AZURE (BIG 01/04) randomised open-label phase 3 trial. Lancet Oncol 2014;15:997-1006.

24. Wong MH, Pavlakis N. Optimal management of bone metastases in breast cancer patients. Breast Cancer (Dove Med Press) 2011;3:35-60.

25. Paterson AHG, Anderson SJ, Lembersky BC et al. Oral clodronate for adjuvant treatment of operable breast cancer (National Surgical Adjuvant Breast and Bowel Project protocol B-34): a multicentre, placebo-controlled, randomised trial. Lancet Oncol 2012;13:734-42.

26. Network NCC. NCCN Clinical Practice Guidelines in Oncology Breast Cancer (Version 1.2018). Available online: https://www.nccn.org/professionals/physician_gls/ pdf/breast.pdf

27. Gnant M, Cle'zardin P. Direct and indirect anticancer activity of bisphosphonates: a brief review of published literature. Cancer Treat Rev 2012;38:407-15.

28. Solomayer EF, Gebauer G, Hirnle P, et al. Influence of zoledronic acid on disseminated tumor cells in primary breast cancer patients. Ann Oncol 2012;23:2271-7.

29. Clezardin P. Bisphosphonates' antitumor activity: an unravelled side of a multifaceted drug class. Bone 2011;48:71-9.

30. Fournier PG, Stresing V, Ebetino FH, et al. How do bisphosphonates inhibit bone metastases in vivo? Neoplasia 2010;12:571-8.

31. Coleman R, Gnant M, Morgan G, et al. Effects of bonetargeted agents on cancer progression and mortality. J Natl Cancer Inst 2012;104:1059-67.

32. Hortobagyi GN, Van Poznak C, Harker WG, et al. Continued Treatment Effect of Zoledronic Acid Dosing Every 12 vs 4 Weeks in Women With Breast Cancer Metastatic to Bone: The OPTIMIZE-2 Randomized Clinical Trial. JAMA Oncol 2017;3:906-12.

33. Amadori D, Aglietta M, Alessi B, et al. Efficacy and safety of 12-weekly versus 4-weekly zoledronic acid for prolonged treatment of patients with bone metastases from breast cancer (ZOOM): a phase 3, open-label, randomised, non-inferiority trial. Lancet Oncol 2013;14:663-70.

34. Shapiro CL, Moriarty JP, Dusetzina S, et al. CostEffectiveness Analysis of Monthly Zoledronic Acid, Zoledronic Acid Every 3 Months, and Monthly Denosumab in Women With Breast Cancer and Skeletal 
Metastases: CALGB 70604 (Alliance). J Clin Oncol 2017;35:3949-55.

35. Gül G, Sendur MA, Aksoy S, et al. A comprehensive review of denosumab for bone metastasis in patients with solid tumors. Curr Med Res Opin 2016;32:133-45

36. Gnant M, Pfeiler G, Dubsky PC, et al. Adjuvant denosumab in breast cancer (ABCSG-18): a multicentre, randomised, double-blind, placebo-controlled trial. Lancet 2015;386:433-43.

37. Gnant M, Pfeiler G, Steger GG, et al. Adjuvant denosumab in postmenopausal patients with hormone receptor-positive breast cancer (ABCSG-18): disease-free survival results from a randomised, double-blind, placebocontrolled, phase 3 trial. Lancet Oncol 2019;20:339-51.

38. Coleman RE, Finkelstein D, Barrios CH, et al. Adjuvant denosumab in early breast cancer: First results from the international multicenter randomized phase III placebo controlled D-CARE study. J Clin Oncol 2018;36:abstr 501.

39. Stopeck AT, Lipton A, Body JJ, et al. Denosumab compared with zoledronic acid for the treatment of bone metastases in patients with advanced breast cancer: a randomized, double-blind study. J Clin Oncol 2010;28:5132-9.

40. Müller A, Templeton AJ, Hayoz S, et al. Incidence of hypocalcemia in patients with metastatic breast cancer under treatment with denosumab: A non-inferiority phase III trial assessing prevention of symptomatic skeletal events (SSE) with denosumab administered every 4 weeks versus every 12 weeks: SAKK 96/12 (REDUSE) (abstract). In: Proceedings of the 2018 San Antonio Breast Cancer Symposium; 2018 Dec 4-8; San Antonio, TX. Philadelphia (PA): AACR; Cancer Res 2019;79:Abstract nr P1-18-01.

41. Hoff AO, Toth BB, Altundag K, et al. Frequency and risk factors associated with osteonecrosis of the jaw in cancer patients treated with intravenous bisphosphonates. J Bone Miner Res 2008;23:826-36.

42. Saad F, Brown JE, Van Poznak C, et al. Incidence, risk factors, and outcomes of osteonecrosis of the jaw: integrated analysis from three blinded active-controlled phase III trials in cancer patients with bone metastases. Ann Oncol 2012;23:1341-7.

43. Van Poznak CH, Temin S, Yee GC, et al. American Society of Clinical Oncology executive summary of the clinical practice guideline update on the role of bone modifying agents in metastatic breast cancer. J Clin Oncol 2011;29:1221-7.
44. Lipton A, Fizazi K, Stopeck AT, et al. Superiority of denosumab to zoledronic acid for prevention of skeletal related events: a combined analysis of 3 pivotal, randomised, phase 3 trials. Eur J Cancer 2012;48:3082-92.

45. Wilkinson GS, Baillargeon J, Kuo YF, et al. Atrial fibrillation and stroke associated with intravenous bisphosphonate therapy in older patients with cancer. J Clin Oncol 2010;28:4898-905.

46. Tanvetyanon T, Stiff PJ. Management of the adverse effects associated with intravenous bisphosphonates. Ann Oncol 2006;17:897-907.

47. Anastasilakis AD, Toulis KA, Goulis DG, et al. Efficacy and safety of denosumab in postmenopausal women with osteopenia or osteoporosis: a systematic review and a metaanalysis. Horm Metab Res 2009;41:721-9.

48. Tomblyn $M$. The role of bone-seeking radionuclides in the palliative treatment of patients with painful osteoblastic skeletal metastases. Cancer Control 2012;19:137-44.

49. McEwan AJB. Palliation of bone pain. In: Murray IPC, Ell PJ. editors. Nuclear Medicine in Clinical Diagnosis and Treatment. Edinburgh, Churchill Livingston, 1994:877-92.

50. Serafini AN. Therapy of metastatic bone pain. J Nucl Med 2001;42:895-906.

51. Fuster D, Herranz R, Vidal-Sicart S, et al. Usefulness of strontium-89 for bone pain palliation in metastatic breast cancer patients. Nucl Med Commun 2000;21:623-6.

52. Liepe K, Franke WG, Koch R, et al: Comparison of rhenium-188, rhenium-186 and strontium in palliation of painful bone metastases. Nuklearmedizin 2000;39:146-51.

53. Krishnamurthy GT, Krishnamurthy S. Radionuclides for metastatic bone pain palliation: a need for rational re-evaluation in the new millenium. J Nucl Med 2000;41:688-91.

54. Collins C, Eary JF, Donaldson G, et al. Samarium-153 EDTMP in bone metastases of hormone refractory prostate carcinoma: a phase I/II trial. J Nucl Med 1993;34:1839-44.

55. Resche I, Chatal JF, Pecking, et al. A dose controlled study of Sm-153 ethylenediaminetetramethylenephosphonate in the treatment of patients with painful bone metastases. Eur J Cancer 1997;33:1583-91.

56. Fischer M, Kampen WU. Radionuclide Therapy of Bone Metastases. Breast Care 2012;7:100-7.

57. Kasalický J, Kraská V. The effect of repeated strontium-89 chloride therapy in bone pain palliation in patients with skeletal cancer metastases. Eur J Nucl Med 1998;25:1362-7. 


\section{Page 10 of 11}

58. Vandyke K, Dewar AL, Farrugia AN, et al. Therapeutic concentrations of dasatinib inhibit in vitro osteoclastogenesis. Leukemia 2009;23:994-7.

59. Schot AF, Barlow WE, Van Poznak CH, et al. Phase II studies of two different schedules of dasatinib in bone metastasis predominant metastatic breast cancer: SWOG S0622. Breast Cancer Res Treat 2016;159:87-95.

60. Hannon RA, Finkelman RD, Clack G, et al. Effects of Src kinase inhibition by saracatinib (AZD0530) on bone turnover in advanced malignancy in a Phase I study. Bone 2012;50:885-92.

61. Wright LE, Frye JB, Lukefahr AL, et al. Curcuminoids block TGF-beta signaling in human breast cancer cells and limit osteolysis in a murine model of breast cancer bone metastasis. J Nat Prod 2013;76:316-1.

62. Yamaguchi $M$, Zhu S, Zhang S, et al. Curcumin analogue UBS109 prevents bone loss in breast cancer bone metastasis mouse model: involvement in osteoblastogenesis and osteoclastogenesis. Cell Tissue Res 2014;357:245-52.

63. Bayet-Robert M, Kwiatkowski F, Leheurteur M, et al. Phase I dose escalation trial of docetaxel plus curcumin in patients with advanced and metastatic breast cancer. Cancer Biol Ther 2010;9:8-14.

64. Ross MH, Esser AK, Fox GC, et al. Bone-Induced Expression of Integrin beta3 Enables Targeted Nanotherapy of Breast Cancer Metastases. Cancer Res 2017;77:6299-312.

65. Pennisi A, Ling W, Li X, et al. Consequences of daily administered parathyroid hormone on myeloma growth, bone disease, and molecular profiling of whole myelomatous bone. PLoS One 2010;5:e15233.

66. Tella SH, Kommalapati A, Correa R. Profile of Abaloparatide and Its Potential in the Treatment of Postmenopausal Osteoporosis. Cureus 2017;9:e1300.

67. Siemionow K, Lieberman IH. Vertebral augmentation in osteoporosis and bone metastasis. Curr Opin Support Palliat Care 2007;1:323-7.

68. Cardoso F. Senkus E, Costa A, et al. 4th ESO-ESMO International Consensus Guidelines for Advanced Breast Cancer (ABC 4). Ann Oncol 2018;29:1634-57.

69. Feyer P, Steingraeber M. Radiotherapy of Bone Metastasis in Breast Cancer Patients - Current Approaches. Breast Care (Basel) 2012;7:108-12.

70. D'Oronzo S, Brown J, Coleman R. The role of biomarkers in the management of bone-homing malignancies. J Bone Oncol 2017;9:1-9.

71. Wada N, Fujisaki M, Ishii S, et al. Evaluation of bone metabolic markers in breast cancer with bone metastasis.
Rossi et al. The treatment of bone metastases from breast cancer

Breast Cancer 2001;8:131-7.

72. G. Oremek, H. Sauer-Eppel, M. Klepzig. Total procollagen type 1 amino-terminal propeptide (total $\mathrm{P} 1 \mathrm{NP})$ as a bone metastasis marker in gynecological carcinomas. Anticancer Res 2007;27:1961-2.

73. Elfar GA, Ebrahim MA, Elsherbiny NM, et al. Validity of osteoprotegerin and receptor activator of NF- $\mathrm{kB}$ ligand for detection of bone metastasis in breast cancer. Oncol Res 2017;25:641-50.

74. Coleman RE, Major P, Lipton A, et al. Predictive value of bone resorption and formation markers on cancer patients with bone metastases receiving the bisphosphonate zoledronic acid. J Clin Oncol 2005;23:4925-35.

75. Brown JE, Cook RJ, Lipton A, et al. Prognostic factors for skeletal complications from metastatic disease in breast cancer. Breast Cancer Res Treat 2010;123:767-79.

76. Lipton A, Cook R, Saad F, et al. Normalization of bone markers is associated with improved survival in patients with bone metastases from solid tumours and elevated bone resorption receiving zoledronic acid. Cancer 2008;113:193-201.

77. Lipton A, Cook R, Brown J, et al. Skeletal-related events and clinical outcomes in patients with bone metastases and normal levels of osteolysis: exploratory analyses. Clin Oncol (R Coll Radiol) 2013;25:217-26.

78. Coleman RE, Lipton A, Costa L, et al. Possible survival benefits from zoledronic acid treatment in patients with bone metastases from solid tumours and poor prognostic features - an exploratory analysis of placebo-controlled trials. J Bone Oncol 2013;2:70-6.

79. Coleman RE, Costa L, Saad F, et al. Consensus on the utility of bone markers in the malignant bone disease setting. Crit Rev Oncol/Hematol 2011;80:411-32.

80. Savci-Heijink CD, Halfwerk H, Koster J, et al. A novel gene expression signature for bone metastasis in breast carcinomas, Breast Cancer Res Treat 2016;156:249-59.

81. Nutter F, Holen I, Brown HK, et al. Different molecular profiles are associated with breast cancer cell homing compared with colonization of bone: evidence using a novel bone-seeking cell line. Endocr Relat Cancer 2014;21:327-41.

82. Holen I, Lefley DV, Francis SE, et al. IL-1 drives breast cancer growth and bone metastasis in vivo. Oncotarget 2016;7:75571-84.

83. Bellanger A, Donini CF, Vendrell JA, et al. The critical role of the ZNF217 oncogene in promoting breast cancer metastasis to the bone. J Pathol 2017;242:73-89. 
84. Westbrook JA, Cairns DA, Peng J, et al. CAPG and GIPC1: BC biomarkers for bone metastasis development and treatment. J Natl Cancer Inst 2016;108:djv360.

85. Fateh-Moghadam A, Stieber P. Sensible use of tumour markers 2nd ed. In: Fateh-Moghadam A. editor. Jürgen

Cite this article as: Rossi L, Longhitano C, Kola F, Del Grande M. State of art and advances on the treatment of bone metastases from breast cancer: a concise review. Chin Clin Oncol 2020;9(2):18. doi: 10.21037/cco.2020.01.07
Hartmann Verlag GmbH, Munich. New York: Wiley, 1993:20-38.

86. Lipton A. Biochemical bone markers in breast cancer. Cancer Treat Rev 2006;32 Suppl 1:20-2. 